\begin{tabular}{|l|c|c|c|}
\hline Eiszeitalter u. Gegenwart & $\mathbf{4 5}$ & $93-108$ & Hannover 1995 \\
\hline
\end{tabular}

\title{
Die Auswirkung alter Kryomere auf die „Rodados Patagónicos“ in Nordpatagonien, Argentinien
}

\author{
Dario Trombotto \& Ana-Lía AhumadA*)
}

Plio-Pleistocene, structure studies, Rodados Patagónicos, paleoenvironment, Puerto Madryn, Northern Patagonia

\begin{abstract}
Kurzfassung: Es wurden Profile von patagonischen Geröllen, einem fluvialen Konglomerat, in der Nähe der Stadt Puerto Madryn bei $43^{\circ}$ s. B. in Nordpatagonien, Argentinien, untersucht, um Gefrier- und Auftaustrukturen zu analysieren. Das Konglomerat stammt aus dem Pliozän/Pleistozän. Gefügeanalysen unterstützen eine Syngenese von kalten Phänomenen und der Ablagerung der patagonischen Gerölle. In den patagonischen Geröllen finden sich syngenetische und epigenetische fossile Eiskeile, die bis ins Tertiär reichen. Es gibt keine absolute Datierung, aber die kryogenen Strukturen lassen verschiedene kalte Perioden vermuten. Sie wurden unter dem Namen Penfordd Kryomer zusammengefaßt. Die paläoklimatische Rekonstruktion ergibt Permafrost und eine Jahresdurchschnittstemperatur, die im Vergleich zu heute $16^{\circ}-17^{\circ} \mathrm{C}$ niedriger war. Die patagonischen Gerölle sind von einem Karbonatnetz mit unterschiedlichen Kalkrettypen durchzogen, das ein orthogonales System erkennen läßt. Während solide Karbonatteile die s. g. Nougat-Struktur (Säulen oder horizontale/quasihorizontale Schichten) bilden, werden die s. g. Fenster von Sektoren ohne Karbonat oder mit nur pulverigem Karbonat gebildet. Die Kalkreste bestehen vorwiegend aus $\mathrm{CaCO}_{3}$, Quarz und vulkanischem Glas. Die Untersuchungen des Gefüges und der Sedimente ergeben folgende paläoklimatische Bedingungen: Aridität, Vulkanismus und kalte Kryomere.
\end{abstract}

\section{[How old cryomeres affected the "Rodados Patagónicos" (patagonian gravel) in northern Patagonia, Argentina]}

\begin{abstract}
The present study analyses different profiles with structures of freezing and thawing which affected the "Rodados Patagónicos" in the region of Puerto Madryn, Northern Patagonia. The "Rodados Patagónicos" are of fluvial origin and are assigned to the Plio-Pleistocene. Fabricstudies support the hypothesis of a synchronization of certain cold events and some deposition-cycles of the "Rodados Patagónicos". The ice wedge-casts may be of syngenetic as well as epigenetic origin and correspond to the cold episodes which belong to the Penfordd Cryomere. As far as
\end{abstract}

\footnotetext{
*) Anschriften der Verfasser: D. Trombotto, Centro Nacional Patagónico (Cenpat), Boulevard Brown 3000, 9120 Puerto Madryn, Chubut, Argentina.

A.-L. Ahumada, Instituto de Sedimentologia, Fundación Miguel Lillo, Miguel Lillo 251, 4000 San Miguel de Tucumán.
}

paleoenvironment is concerned, the structures mentioned above required a permanently frozen ground, which is to say a mean annual temperature of at least $-4^{\circ} \mathrm{C}$, indicating a temperature increase of nearly $17^{\circ} \mathrm{C}$ since formation time. The characteristic heterogeneity of the profiles can be described as a system of "columns", subhorizontal levels and layers of impregnation forming a "nougat-structure", composed mainly of $\mathrm{CaCO}_{3}$, volcanic glass and quartz. The structures reveal phenomena of leakage and freezing in the fluvial conglomerate. The analyses of the clast-fabric and architecture of the sites allow to recognize structures and reconstruct the paleoenvironment from the Neogene on; an environment with cold impulses, an important volcanic activity and particularly arid or semiarid climatic conditions alternating with the deposition of the "Rodados $\mathrm{Pa}$ tagónicos" as outstanding features.

\section{Einleitung}

Der Ursprung der "Rodados Patagónicos" oder patagonischen Gerölle (im folgenden RP genannt) wurde von zahlreichen Autoren zu verschiedenen Zeiten diskutiert. Es wird betont, daß bei ihrer Entstehung, Distribution und Ablagerung an verschiedenen Orten in Patagonien diverse exogene Agentien (fluviale, fluvioglaziale, an der Küste mit Beteiligung des Meeres) eine Rolle gespielt haben. Feruglio (1950) und Auer (1956) fassen die Hypothesen und Theorien der verschiedenen Autoren zusammen. Caldenius (1940) erwähnt die Beteiligung der Solifluktion zusammen mit Denudationszyklen während der Kryomere im Osten Patagoniens. Kryomere i. S. von LütTig (1965) sind die kalten Perioden des Quartärs.

Man kann mit Gewißheit sagen, daß intensive Kälteperioden, Vulkanismus und Aridität Patagonien regelmäßig seit der Erhebung der "Cordillera de los Andes" heimgesucht haben. Die episodischen Kälteperioden haben nicht nur zu Vergletscherungen in der Kordillere geführt, sondern das Eis erreichte in Südpatagonien während der großen Vergletscherung den Atlantik (vor ca. 1,2 Mio. Jahren; MERcer 1976) "Glazoblasten“ (Groeber 1950) oder lokale Vergletscherungen ohne größere subregionale Aus- 
breitung sind bis heute ungeklärt. Seit dem Messinian zeugt die südliche Kordillere von Vergletscherungen (MERCER \& SUTTER 1982). Die sedimentären Eigenschaften des Rionegrense belegen bereits die kalten und trockenen klimatischen Bedingungen und die stets vorhandene vulkanische Aktivität. Die paläoklimatischen Bedingungen während der Kryomere waren von sich verstärkenden Winden aus westlicher Richtung begleitet, die die Aridität in den mittleren Breitengraden Argentiniens noch steigerten (Gonzales \& TrombotTo 1990).

Die RP als Formation mit ihren Entstehungseigenschaften enthalten, wie im folgenden gezeigt wird, eindeutig Sedimente fluvialen Ursprungs, die beweisen, daß das Klima nicht nur an den Anden, sondern in ganz Patagonien zeitweilig feucht gewesen sein muß. Zeitpunkte größerer Feuchtigkeit sowie das Schmelzen der Eismassen waren an der Distribution und Akkumulation der RP seit dem Plio-Pleistozän beteiligt. Die kryogenen Strukturen in den RP-Ablagerungen zeigen auch, daß sie Gefrier- und Auftauzyklen unterworfen waren. Die Auswirkungen der Gefrier- und Auftauzyklen, fossile Eiskeile und das zwangsläufige Vorhandensein von Permafrost im Zusammenhang mit den RP wurden für Nordpatagonien von Auer (1956 u. 1970), CZAJKA (1955) und
LIss (1969) beschrieben. 1981 wurde die geologische Karte von Puerto Madryn ( 43 h) veröffentlicht, in der Haller die Froststrukturen dem Kryoturbationsprozeß zuordnet, der zeitlich vor oder zu Beginn der Ablagerung der RP liegt. Später wurden diese Strukturen paläopedologisch betrachtet (DeL VALLE \& BELTRAMONE 1987) und als Phänomene des letzten Hochglazials eingestuft (CORTE \& Beltramone 1984; Beltramone 1989; Corte 1991). Vogt (1990) untersuchte chemische Ausfällungen, die mit diesen kryogenen Formen zusammenhängen. TRомвотто (1992) faßte die Gesamtheit der kalten Phänomene unter dem lokalen Namen „Penffordd Kryomer" (Penfordd, aus dem Walisischen: Penfordd; Hauptweg von Puerto Madryn ins Tal des Flusses Chubut) zusammen, einer Vielzahl vorzeitlicher kryogener Prozesse, die sich während und nach der Ablagerung der RP in der Zone von Puerto Madryn ereigneten.

Die Zielsetzungen dieser Arbeit sind:

a) die kryogenen Formen zu untersuchen,

b) anhand bewährter Techniken die Strukturen, die die Gefrier- und Auftauzyklen in den RP hervorgerufen haben zu bestimmen,

c) die paläoklimatische Vergangenheit zu rekonstruieren und dem entsprechenden sedimentären Rahmen zuzuordnen.

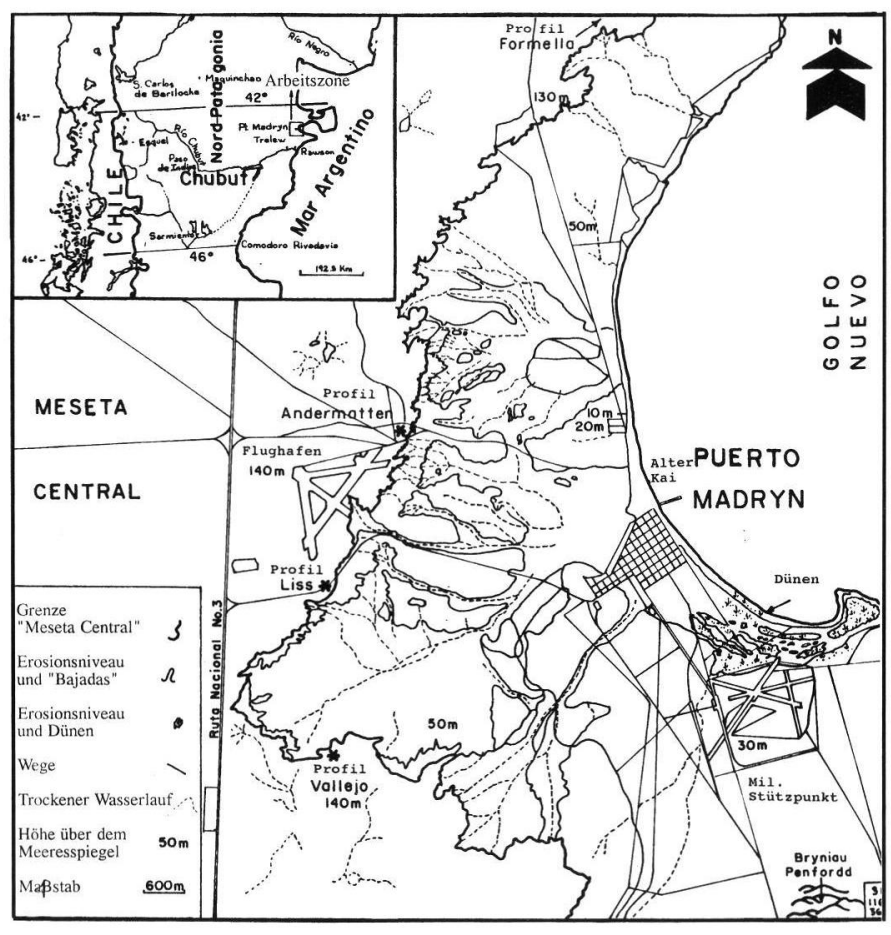

Abb. 1: Karte des Untersuchungsgebietes mit Hilfe von Luftbildaufnahmen (1969 - 71) erstellt. Maßstab: 1:60.000. Zwischen Profil Liss und Profil Vallejo: „Bajo Grande"

Fig. 1: Map of the study area according to satellite images (1969-71). Scale: 1:60.000. Between profile Liss and Vallejo: "Bajo Grande".

\section{Untersuchungsgebiet}

Das Untersuchungsgebiet umfaßt die nähere Umgebung der Stadt Puerto Madryn $42^{\circ} 47^{\prime}$ südl. Breite und $65^{\circ} 65^{\prime}$ westl. Länge (Abb. 1) und säumt die sogenannte „Meseta Central“. Letztere reicht fast bis an den Atlantik, erkenntlich an der charakteristischen Landschaft der MicroBadlands, die vor allem aus dem Patagoniano (marines Oligozän/Miozän) und Sedimenten des Neogens entstanden sind. Die Sedimente der Formation „Puerto Madryn“ (HALLER 1981) sind dem Neogen zuzuordnen: „Entrerriense“ und „Rionegrense" bestehen aus marinen Sedimenten, aus tiefen und seichten Gewässern, sowie aus kontinentalen Sedimenten und enthalten zahlreiche Fossilien (Wirbeltiere, wirbellose Tiere und Pflanzenreste).

Die Tektonik wird anhand regionaler NE-SW Strukturen und Variationen im lithologischen Profil erkennbar. Die nordpatagonische Küste hebt sich, allerdings in unterschiedlichen Maßen je nach geographischer Lage (CODIGNOTTO et al. 1991). 


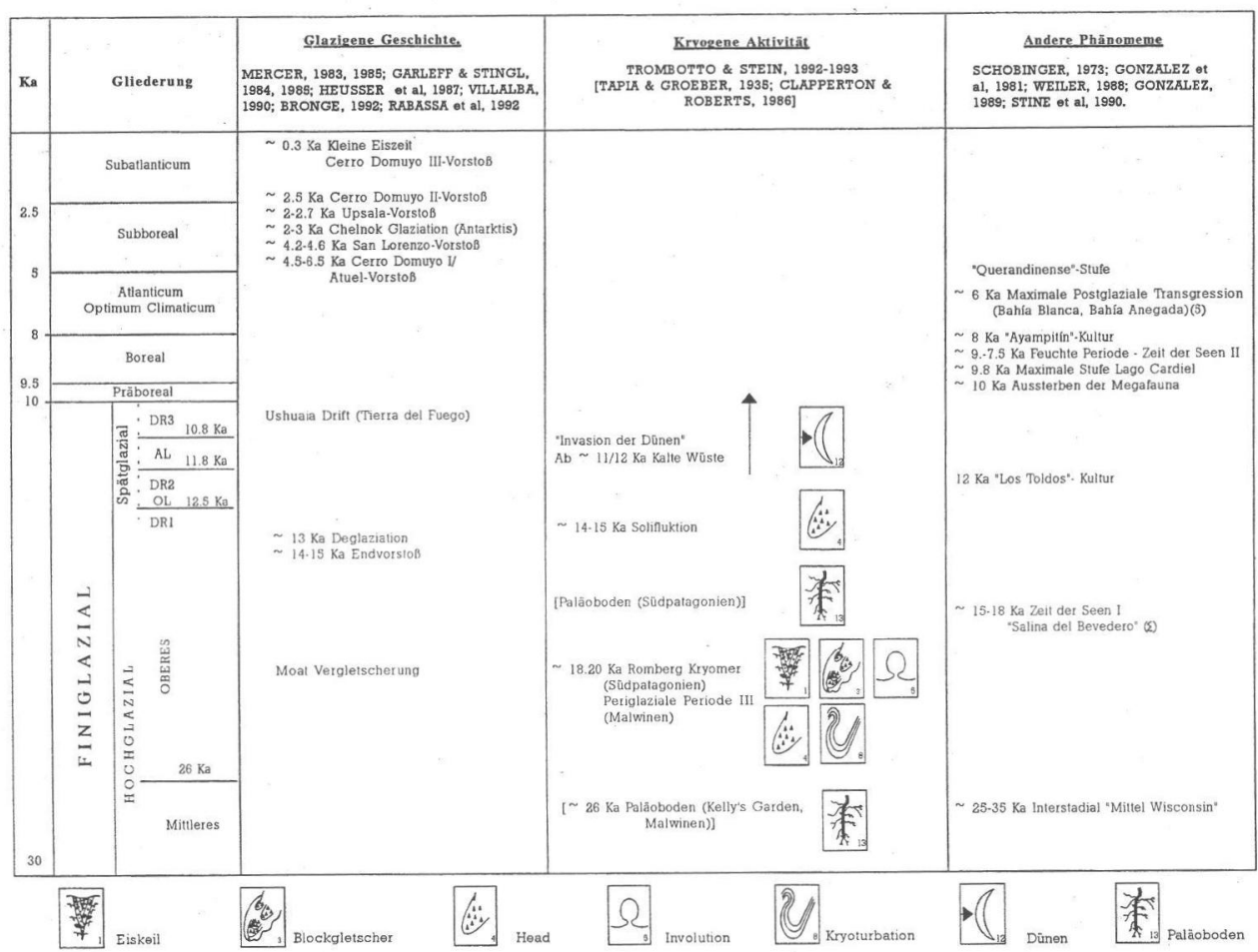

(5) verschiedene, ( $\Sigma$ ) zahlreiche Niveaus

In der Nähe der Ausläufer der Meseta zeichnen sich sanfte Hügel ab, die „Bryniau Penfordd“ (Bryniau = plural von "Bryn" = Hügel in walisischer Sprache), die zweifellos Relikte der Denudationszyklen mit periglazialem Einfluß darstellen.

Die durchschnittliche Jahrestemperatur im Untersuchungsgebiet liegt bei $13,6^{\circ} \mathrm{C}$ und die durchschnittliche Niederschlagsmenge pro Jahr beträgt $173 \mathrm{~mm}$. Zwischen 1905 und 1950 betrug die maximale registrierte Temperatur $39,7^{\circ} \mathrm{C}$ und das absolute Minimum lag bei $11,6^{\circ} \mathrm{C}$. Der Wind weht vorwiegend aus westlicher Richtung. Nach Troll \& PAFFeN (1969) entspricht das Untersuchungsgebiet einem gemäßigt kühlen Klima und ist als Steppe (D) oder Halbwüste (III, 12a) einzuordnen. Phytogeographisch gehört das Gebiet zur „Provinz Monte“ mit Larrea divaricata, L. Nitida, Prosopidastrum globosum, Bougainvillea spinosa und Condalia microphylla (CABRERA 1976).

Die Tabellen 1 und 2 dokumentieren die kryogene und klimatostratigraphische Geschichte der Region. Tabelle 1 versucht durch die bekannten und datierten glazigenen Ablagerungen eine Korrelation zu den kryogenen Phänomenen herzustellen. Tabelle 2 spezifiziert die letzten $25 \mathrm{ka}$ der Tabelle $1(\mathrm{Ma}=$ Millionen Jahre; $\mathrm{ka}=1000$ Jahre).
Die im Text erwähnten Profile heißen (Abb. 1):

1) Liss

2) Andermatten

3) Vallejo

\section{Quartäres nordpatagonisches Profil}

Auf den erwähnten neogenen Sedimenten (Formation „Puerto Madryn“) lagerten sich unregelmäßig die „Rodados Patagónicos“ (RP) fluvialen Ursprungs und plio-pleistozänen Alters ab. Die RP sind typische patagonische quartäre Sedimente, deren Mächtigkeit sehr variabel ist, je nachdem, ob die Sedimente auf positiven oder negativen Geoformen liegen. Abb. 2 zeigt die komplette stratigraphische Gliederung von zwei Profilen. Die charakteristischen Profile weisen alle eine kontinuierliche Schicht mit nicht allzu großen Geröllen (durchschnittlich ca. 3 - $5 \mathrm{~cm}$ Durchmesser), ein ungeordnetes Gefüge mit einer $\mathrm{CaCO}_{3}$-reichen Matrix (Horizonte zwischen 30 und $55 \%$ ), sowie hauptsächlich Quarz und vulkanisches Glas auf. Die Dicke variiert und kann bis zu $1 \mathrm{~m}$ ausmachen. In Ausnahmefällen kann man an den Seiten einen Paläoboden mit Polyedergefüge, Farbe 8.3 5 YR / 8.4 7.5 YR erkennen, 
Tabelle 2

Table 2

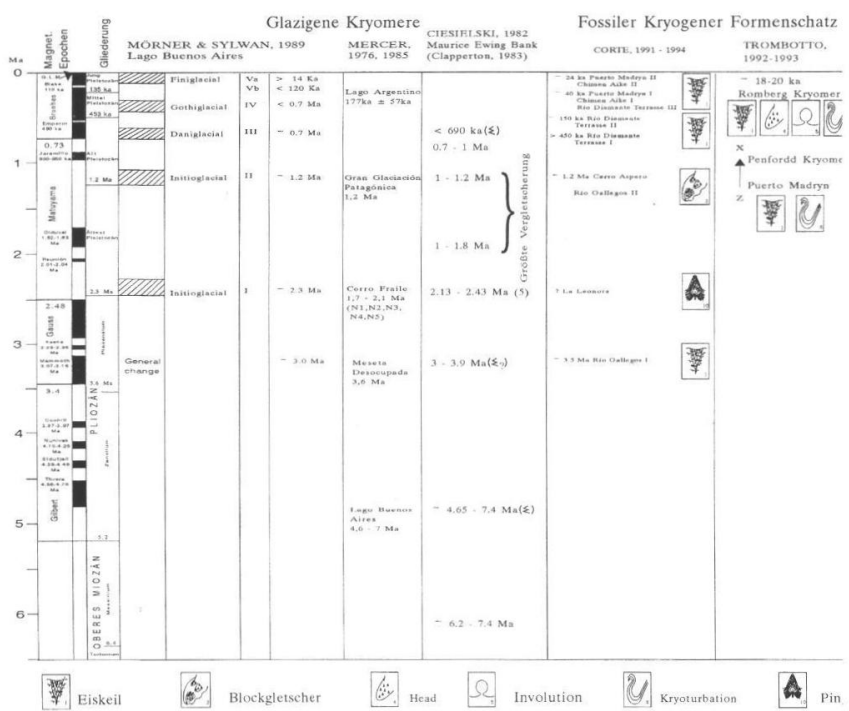

$\underline{\text { Profil Liss A }}$

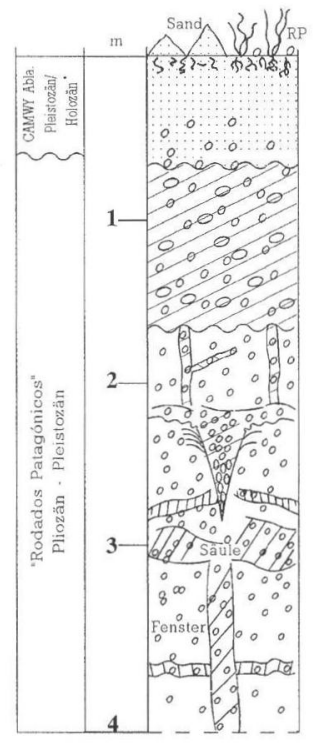

$\underline{\text { Profil Liss B }}$

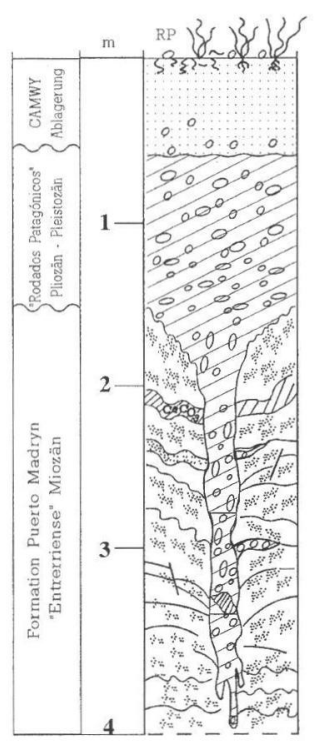

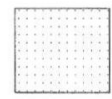

Feiner Sand

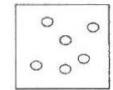

Konglomerat

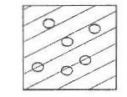

Kalkablagerung

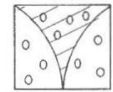

Fossiler Eiskeil

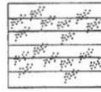

Sandstein

Abb. 2: Profil Liss A und B

Fig. 2: Profile Liss A and B

der reich an $\mathrm{CaCO}_{3}(24 \%)$ ist, einen geringen Gehalt an Ton und einen wesentlichen Anteil an Schluff (26\%) und Sand (36\%) enthält.

Unterhalb dieser Schicht und in den RP befindet sich ein netzartiges System aus „Säulen“, horizontalen und quasihorizontalen Niveaus und „Fenstern“ (Abb. 2, 3 u. 4). Die ersten drei gehen aus der Einbettung der RP in Material des Typs „Tosca“ hervor, welches sich hauptsächlich aus Ca$\mathrm{CO}_{3}$, Quarz, vulkanischem Glas und Feldspat zusammensetzt. Im Gegensatz zu den Säulen ist das Material der Fenster kalkfrei oder kalkarm und pulverig. Sie sind sehr anfällig für Verwitterung (Trombotтo 1992). Die Entstehung des kalkhaltigen Netzes hängt mit dem Versickern von Wasser unter der Oberfläche und mit der Mineralausspülung zusammen. Dieses Netz wird hier ausdrücklich erwähnt, weil es kryogene Strukturen konserviert und eng mit deren Entstehung verknüpft ist. Darüber hinaus erleichtert es das Erkennen dieser Strukturen (Abb. 3).

Mit seinen unterschiedlichen Anteilen an $\mathrm{CaCO}_{3}$ stellt es sowohl horizontal als auch vertikal ein breites Spektrum verschiedener Strukturen dar: von der massiven „Nougatstruktur" (TrombotTo 1992) bis zur pulverigen Struktur der „Fenster“. Die unterschiedlichen Konkretionstypen hängen von der Konzentration der Mineralien und sehr wahrscheinlich vom Alter ab. Sic wurden als a) Nougat-Struktur oder massive Form; b) pulverförmige Struktur; c) Würfelzucker-Struktur; d) streifenartige Kalkstruktur und als e) Krusten charakterisiert. Ein Konkretionstyp tritt beispielsweise in "Zwiebelform" auf, d. h. in konzentrischen siliziumhaltigen Schichten (Trombotто 1992). Oberhalb der RP-Schichten finden sich im allgemeinen äolische Sedimente, die dem Holozän zugeschrieben werden und die sich in einem kalten und ariden Paläoklima abgelagert haben. Diese Ablagerungen werden informell als "Camwy" (Camwy, aus dem Walisischen; Name des Flusses und der Region Chubut) bezeichnet (Trombotтo 1992). Die gra- 


\section{1}

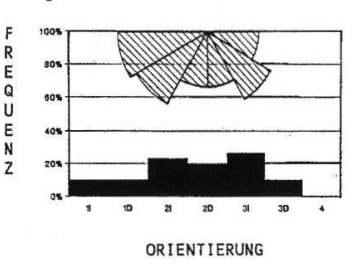

2 Sektor: "Säule"

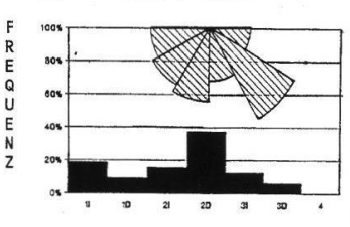

ORIENTIERUNG

3 sektor: "Linkes Fenster"

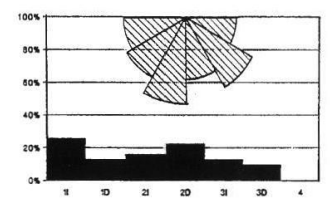

ORIENTIERUNG
4 Sektor: "Nougat"
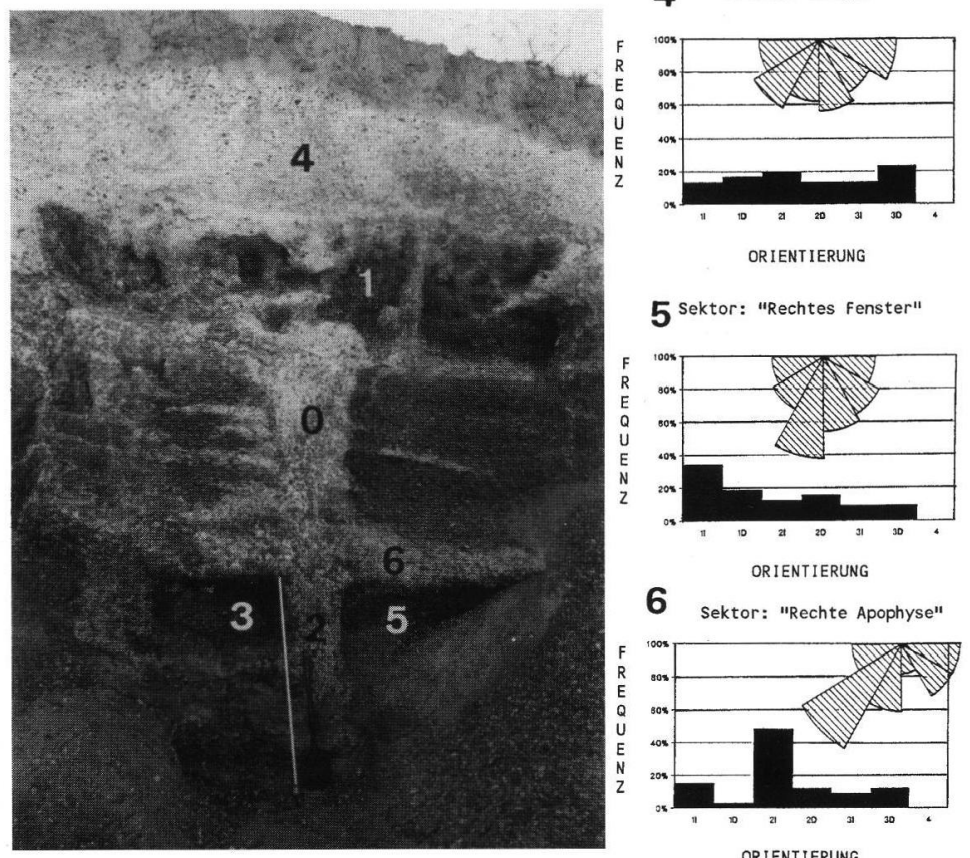

ORIENTIERUNG

5 Sektor: "Rechtes fenster"

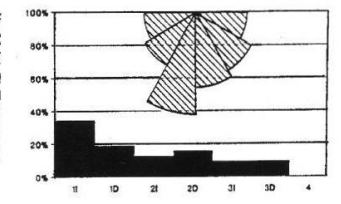

ORIENT IERUNG
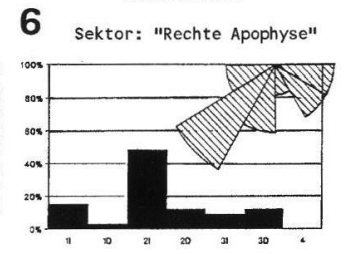

ORIENTIERUNG
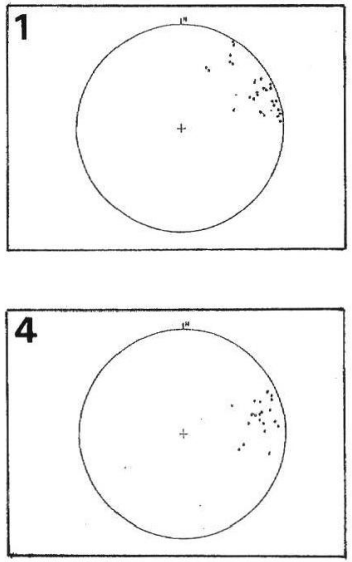
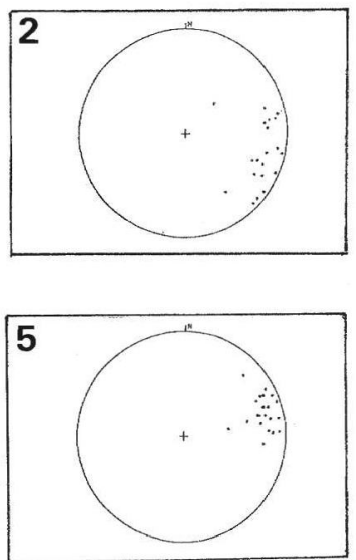
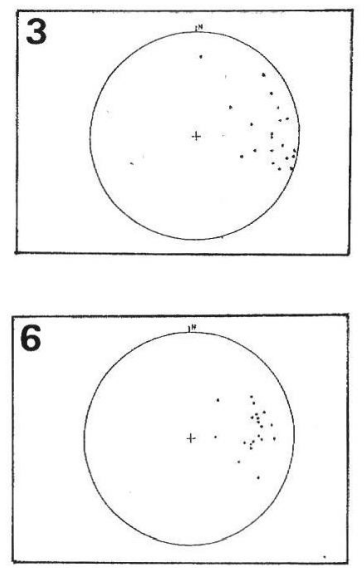

Abb. 3: Profil Liss. Maßstab: $65 \mathrm{~cm}$ (= Länge des Spatens). Die Nummern entsprechen der Numerierung der Diagramme. 0 entspricht Abb. 8.

Fig. 3: Profile Liss. Scale: $65 \mathrm{~cm}$ (= length of the spade). The numbers correspond to those of the diagrams. 0 corresponds to Abb. 8.

nulometrischen Eigenschaften dieser Ablagerungen (z. B. im Profil Liss) weisen einen Mittelwert an sehr feinem Sand auf (39\%), während der Schluffgehalt bei $23 \%$ und der Tongehalt bei $7 \%$ liegen. Fossile Eiskeile und Kryoturbationen finden sich in den RP, und Kryoturbationen treten in den oberen Schichten des Obertertiärs, an der Grenze zu den RP auf. Das Obertertiär wird yon epigenetischen fossilen Eiskeilen durchdrungen (Abb. 6).
Das quartäre Profil Liss hat zwei Typen (Abb. 2). Die Untersuchung des Profils A läßt deutlich Kryoturbation und einen syngenetischen Eiskeil mit folgenden Eigenschaften erkennen:

a - Keilform, nach unten spitz zulaufend;

b - diffuse Deformierung der seitlichen Zonen in der „Säule" und sichtbare Veränderungen und Deformationen nach oben hin und im benachbarten Gestein c - Beziehung zu quasihorizontalen Kalkretschichten; 


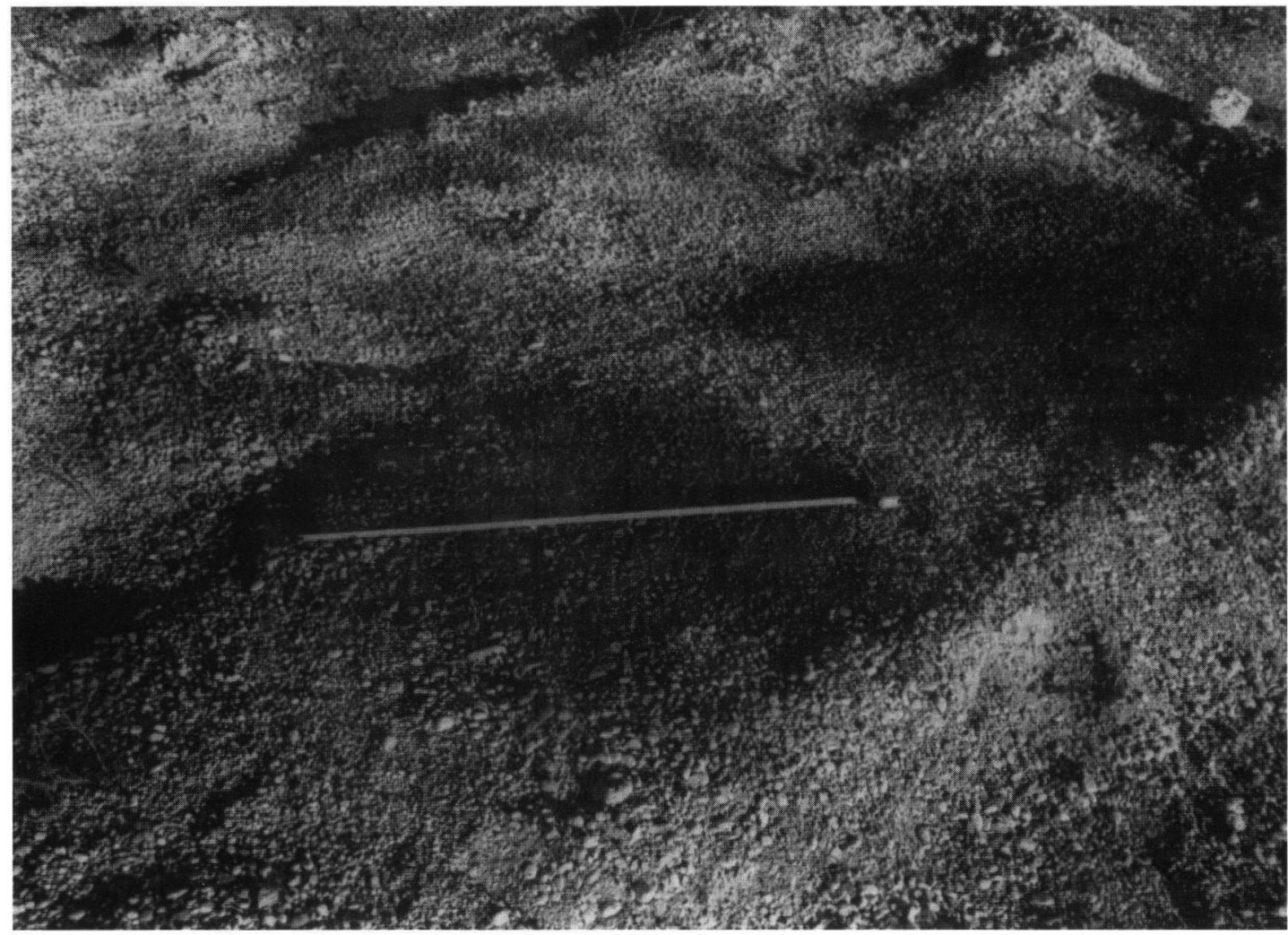

Abb. 4: Profil Liss. Polygonsystem.

Fig. 4: Profile Liss. Polygonal system.

d - Längsachsen des Füllmaterials vertikal;

e - quasivertikale Stratifizierung des Füllmaterials;

f - oberer Teil konkav mit differentieller Erosion;

g - kleine und unregelmäßige Spitze mit der „Säule“ verkrustet (ТRомвотто 1992).

Die genaue Untersuchung eines Profils (Liss, Abb. 1) läßt deutlich die Beziehung zwischen der Granulometrie der RP und einer kalkhaltigen Einbettung erkennen (Abb. 7 und 8). Der Kalk dringt zu verschiedenen Zeiten von oben nach unten durch die Mobilisierung mineralisierbaren Wassers ein. Das läßt sich anhand verschiedener Kalktypen beweisen. Die Kalktypen variieren in Kalkgehalt, Struktur und Härte.

Dieser Transport hängt mit dem ursprünglich offenen Gefüge der fluvialen Ablagerungen zusammen und wurde von den Korngrößenveränderungen der RP besonders von ausgespülten Feinsedimenten z. B. in $80 \mathrm{~cm}$ und $135 \mathrm{~cm}$ Tiefe (Abb. 7 und 8) bedingt. Zeichnet sich in $135 \mathrm{~cm}$ Tiefe möglicherweise die Basis einer Auftauschicht ab? Auch die Beeinflussung der Texturen durch Kryogenese hat zur Ablagerung von Mineralien beigetragen. Unter Kryogenese versteht man die kryogenen Prozesse, die durch Gefrieren und Auftauen von Wasser im Boden, Sedimenten und Steinen typische Strukturen und Texturen hervorrufen. Die so entstandenen Kalkrete verschiedener Art - Formen der Kalkanreicherung, die überwiegend aus Calciumcarbonat bestehen - sind vertikal unterbrochen und verbinden Schichten mit kryogenen Strukturen z. B. syngenetische, fossile Eiskeile (Abb. 5) und werden von anderen Schichten aus RP unterschiedlicher Textur, Struktur und Form bedeckt (Abb. 2, 7, 8 und 3). Ein horizontaler Schnitt durch die RP unterhalb $1,50 \mathrm{~m}$ läßt meistens ein unregelmäßiges Polygonsystem erkennen (Abb. 4).

Während das dreidimensionale Netz der Profile die Fließrichtung mineralisierten Wassers anzeigt wenn wir grob dem Modell der Krackelierung durch Austrocknung folgen -, deuten die „Säulen“ auf Fissuren hin, dort wo mineralisiertes Wasser eingedrungen oder ausgetreten ist. Vermutlich ist es auf diesem Wege auch zu einer weiteren Ausspülung und zum tieferen Vordringen der „Tosca“ gekommen.

Bei den „Fenstern“ liegen die Mediane und Mittelwerte über 2 phi und gehören damit eindeutig zu Kies. Wenn wir von der Kalkkomponente, die die Formen bestimmt, absehen, sind sich die "Säulen" und "Fenster" statistisch gesehen ausgesprochen ähnlich. Allerdings enthalten die „Säulen“ und Apo- 

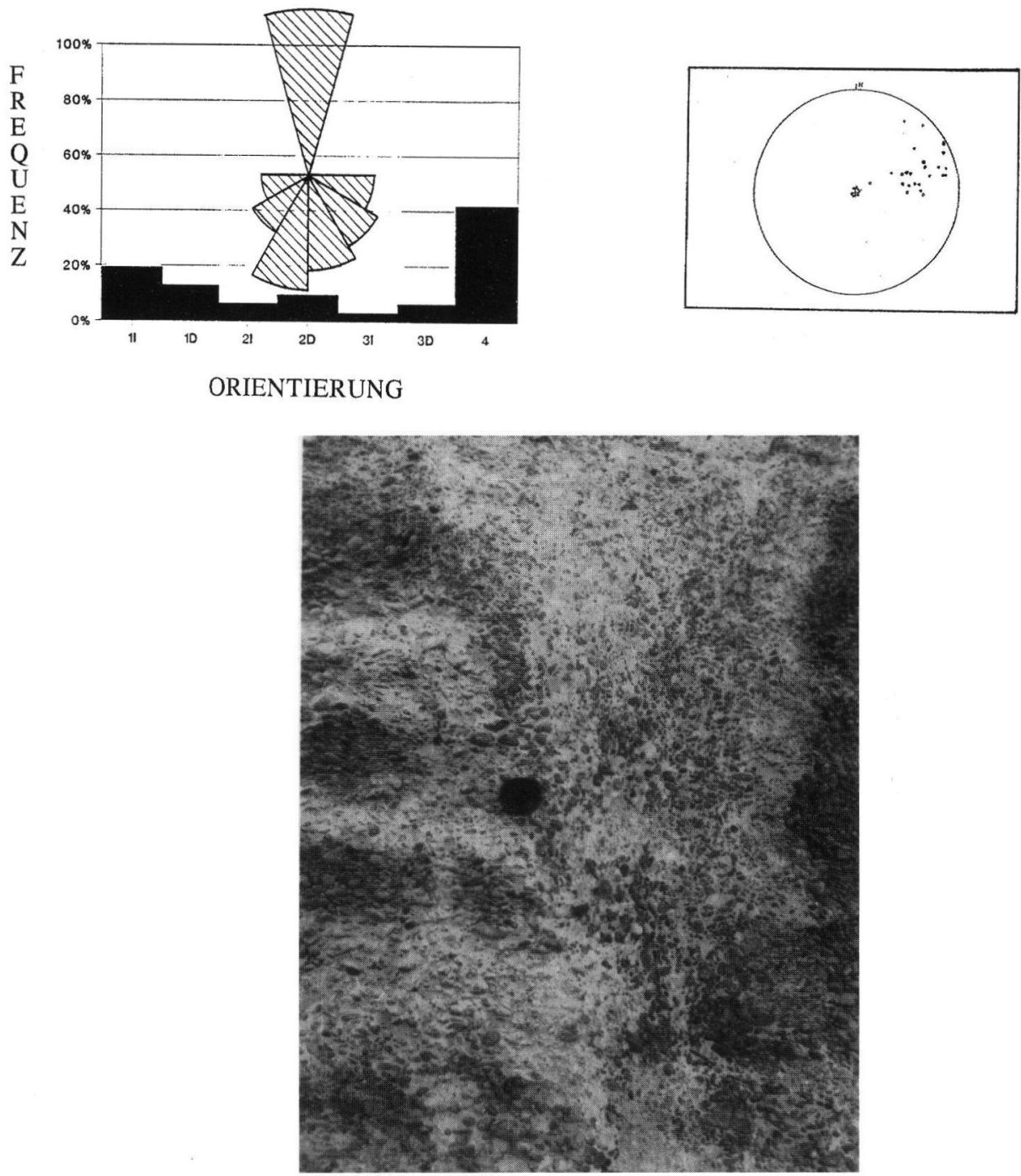

Abb. 5: Profil Liss. Fossiler syngenetischer Eiskeil (siehe Abb. 7). Maßstab: 5,4 cm. Dazugehörige Diagramme: Sitummetrie und Poldiagramm.

Fig. 5: Profile Liss. Syngenetic ice wedge cast (compare photo 1). Scale: $5.4 \mathrm{~cm}$. Including corresponding diagrams: Sitummetry and polediagram.

physen einen beträchtlichen Anteil anf einen Komponenten wie z. B. Feinsand. In den „Fenstern" hingegen sind die Komponenten deutlich reduziert. Sie enthalten praktisch weder Ton noch Schluff, während diese Komponenten in den „Säulen“ und Apophysen mit 2 - $3 \%$ vertreten sind.

Viele der beschriebenen Prozesse: Kryogenese, Krackelierung, Ausspülung, Kalkablagerung etc. haben vermutlich unter der Präsenz von Permafrost und einer Paläo-Auftauschicht stattgefunden. Diese Schicht unterschiedlicher Mächtigkeit scheint die
Zirkulation und die Ausspülung der Mineralien beeinflußt zu haben. Die „Säulen“ sind zum Teil mit fossilen epigenetischen und syngenetischen Eiskeilen assoziiert (Abb. 3 und 5). Man kann davon ausgehen, daß die Gefrier- und Auftauzyklen die $\mathrm{CaCO}_{3}$ Ausfällung gefördert haben (HALlet 1976).

Mit Hilfe absoluter Datierungen $\left({ }^{14} \mathrm{C}\right)$ wurden die Kalkrete der Säule dem Hochglazial zugeordnet (Corte \& Beltramone 1984; Del Valle \& Beltramone 1987; CORTE 1991). Hierbei muß man aber einwenden, daß die Abweichungen sowie vertikal und ho- 


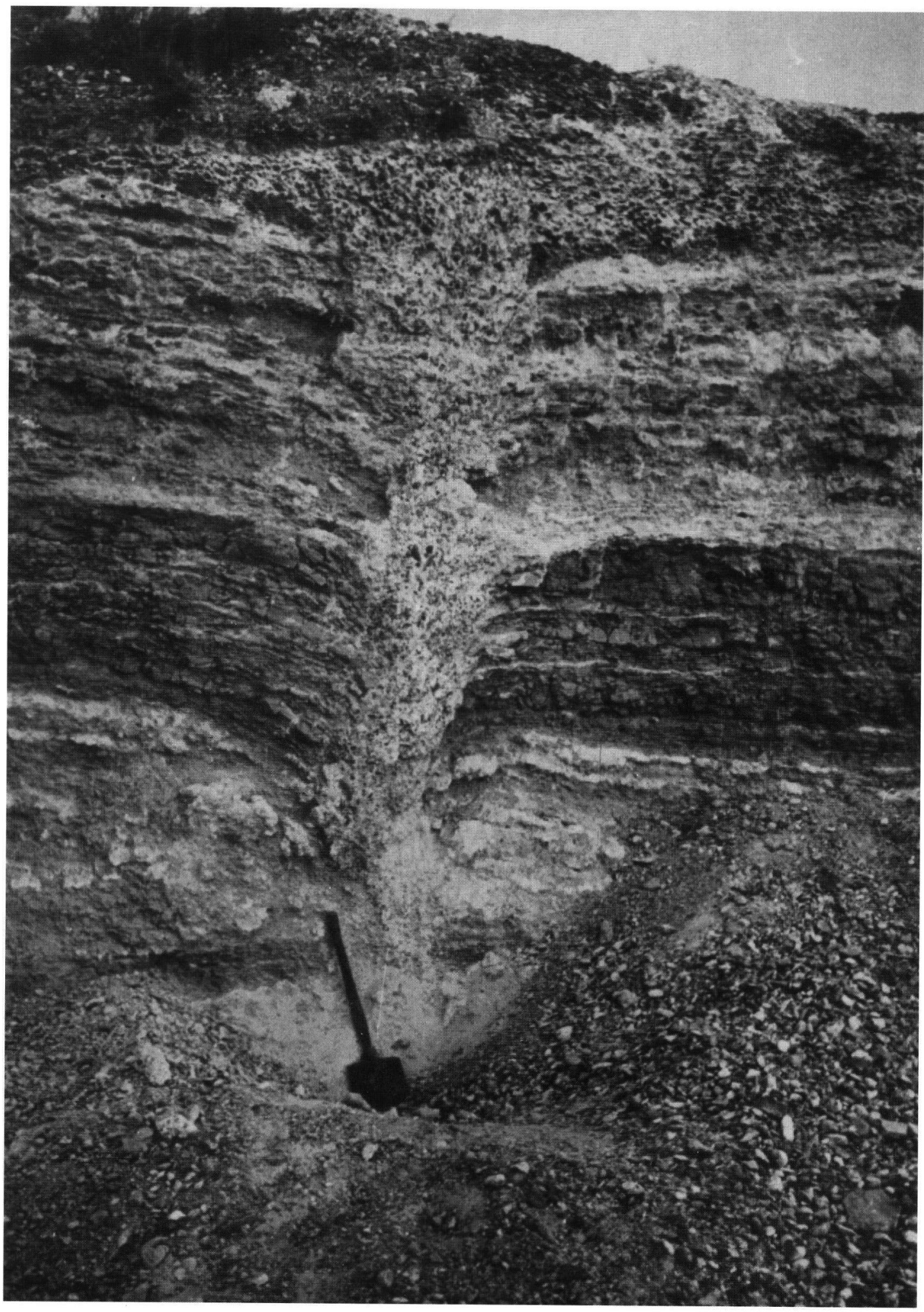




\section{PROFIL LISS 20-H}

\section{Tiefe $(\mathrm{cm})$}

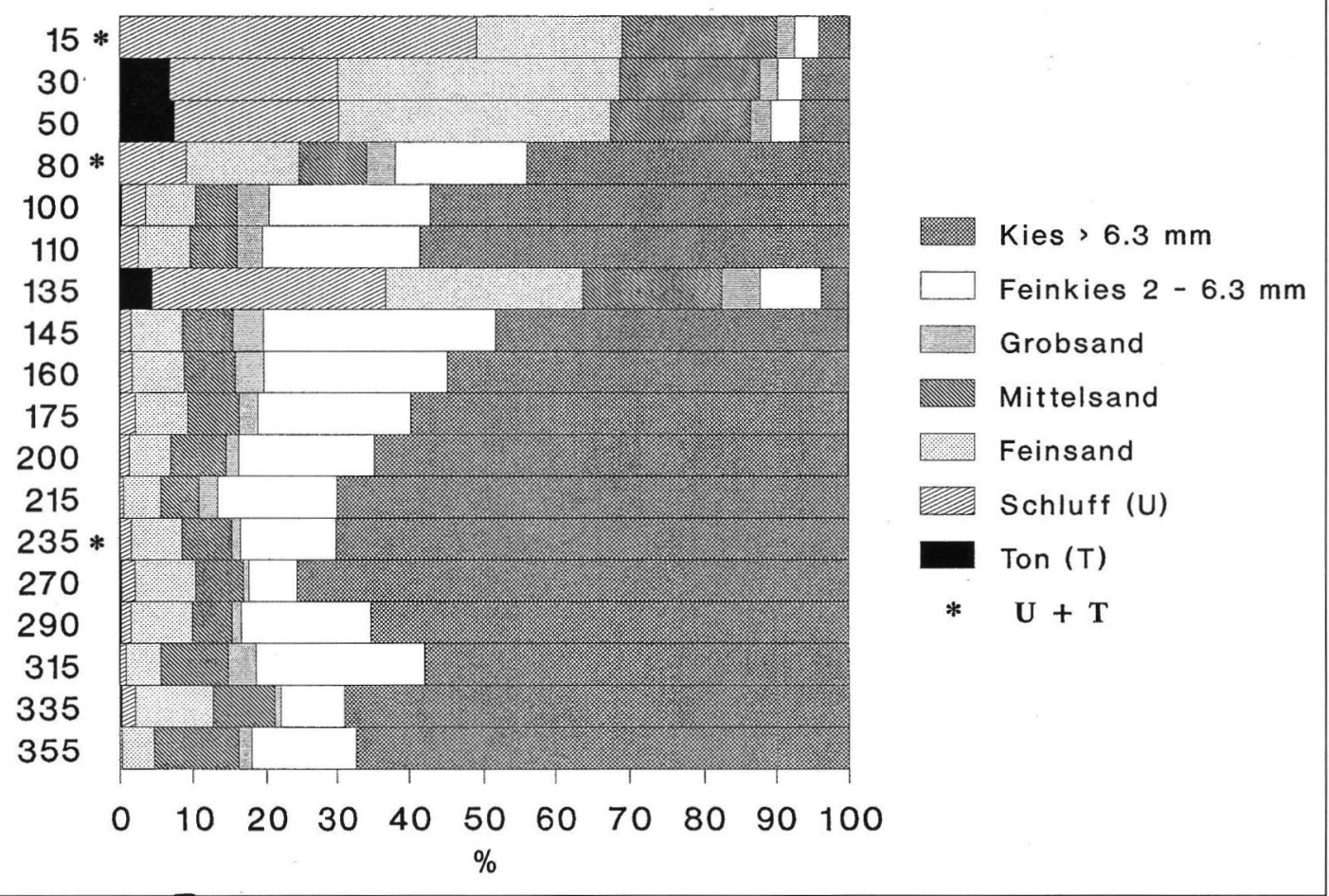

Abb. 7: Profil Liss (A). Granulometrische Prozentanteile.

Fig. 7: Profile Liss (A). Granulometric percentages.

rizontal miteinander verbundene Karbonate die Datierung verfälscht haben könnten. Es gelang jedoch anhand der Datierungen, die Kalkrete dem letzten Karbonatisierungsprozeß zuzuordnen. Die Ablagerung des $\mathrm{CaCO}_{3}$ muß nach der Genese der Strukturen erfolgt sein. Die verschiedenen Kalkrettypen legen jedoch mehrere Kalkablagerungszeitpunkte nahe. Die elektronenmikroskopische Untersuchung der Quarzkornoberflächen bekräftigt die Hypothese einer langwährenden chemischen Aktivität nach der Ablagerung. Die Feinsedimente stammen aus den RP. Das allochthone Material hingegen ist aufgrund der Auswirkung der chemischen Kategorien anhand der Textur der Quarzkornoberfläche nur sehr schwer zu erkennen. Die chemischen Kategorien sind die Mikrotexturen auf den Quarzoberflächen, die durch chemische Aktivität hervorgerufen werden (Auflösung, Silizium-Anlagerung etc.). Das deutet darauf hin, daß das Füllmaterial der fossilen, kryogenen Strukturen sehr alt ist (TRомвотто 1992). Manche Autoren (s. NiLsson 1983) glauben, daß Kar- bonatisierungszyklen auf kalte Perioden hindeuten. In Zusammenhang mit den kryogenen Strukturen und Kryoturbationen, die in Kombination mit den Karbonaten auftreten, könnte hier eine gründliche isotopische Analyse Klarheit schaffen. Die große Ausdehnung der Kalkrete in Patagonien von der Kordillere bis zur Küste läßt vermuten, daß der Wind das ursprünglich kalkhaltige Material in Pulverform transportiert hat (PÉwé 1984). In den „Fenstern“ läßt sich häufig pulveriges oder lockeres Material beobachten.

Die erwähnten Karbonatisierungszeitpunkte müssen mit Austrocknung und hygrischen Impulsen abgewechselt haben, die dazu beigetragen haben, daß das mineralisierbare Wasser die Mineralien in einer semiariden Umwelt mobilisieren konnte. Hängen diese hygrischen Impulse möglicherweise mit der vulkanischen Aktivität zusammen? Aufgrund des Hohlraumgefüges des Alluvions, durch ein Wegenetz und aufgrund der vorhandenen Niederschlagsmenge sind die Kalkrete weiter in die Tiefe vorge- 


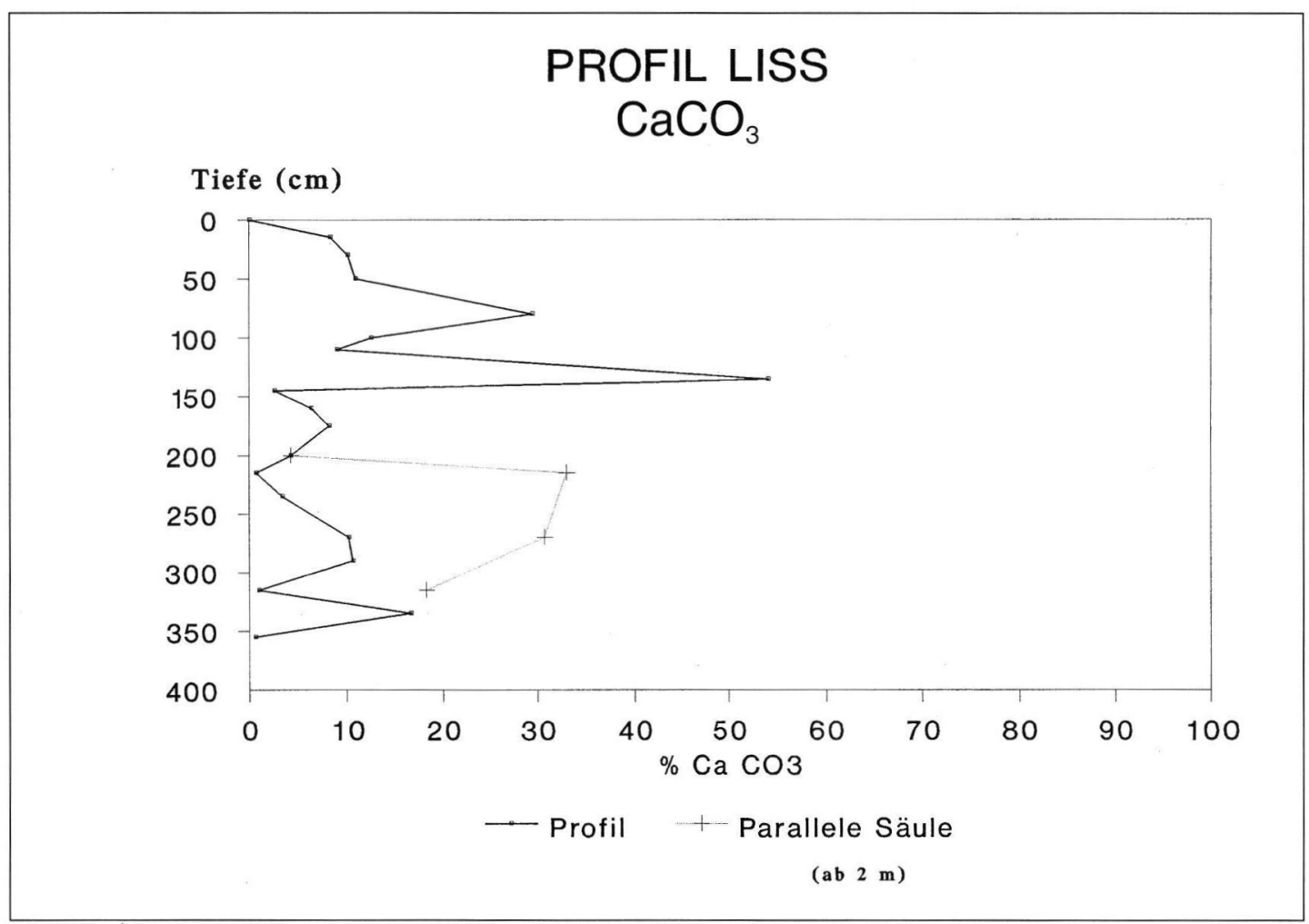

Abb. 8: Profil Liss (A). Prozentanteile $\mathrm{CaCO}_{3}$.

Fig. 8: Profile Liss (A), Percentages of $\mathrm{CaCO}_{3}$.

drungen und haben die bereits bestehenden Ablagerungen modifiziert. Man kann davon ausgehen, daß das mineralisierbare Wasser mit höherem $\mathrm{CO}_{2}$ Gehalt eine größere Ausspülungskapazität besaß und mehr $\mathrm{CaCO}_{3}$ transportieren konnte. Das Vorhandensein großer Mengen an Eruptionsmaterial steht für eine hohe, regelmäßig wiederkehrende vulkanische Aktivität, die von großer Bedeutung ist, da die vulkanischen Gase einen hohen $\mathrm{CO}_{2}$-Gehalt haben und sich auf das Paläoklima ausgewirkt haben können.

\section{Angewandte Methoden}

Für die vorliegende Arbeit wurden zunächst geeignete, repräsentative Orte ausgesucht. Hierbei mußten die Heterogenität der Profile in einer periglazialen Umwelt und die Schwierigkeit, sie in ihrer lateralen Diskontinuität zu studieren, berücksichtigt werden. Die Profile wurden nach geokryologischen Maßstäben angelegt.

Für die Korngrößenverteilungen wurden die traditionellen Methoden angewandt und es wurde feucht, trocken und sowohl feucht als auch trocken gesiebt. Für die Schluffe und Tone wurde die Pipettiermethode angewandt. Die statistischen Daten wurden anhand der Formeln von FOLK \& WARD (PETTijOHN et al. 1987) ermittelt. Einige Profile der RP wurden im Abstand von $15 / 20 \mathrm{~cm}$ und bis zu $4 \mathrm{~m}$ Tiefe beprobt, um Texturen, Abwandlungen und Relationen zu den Kalkablagerungen bestimmen zu können. Für die Poldiagrammanalyse und die Sitummetrie wurde die Methodik benutzt, die von Ahumada \& Trombotтo (1993) und Trombotтo (1990) für aktuelle, periglaziale Milieus entwickelt wurde; in diesem Fall wurde das Wolffsche stereographische Netz benutzt. Letzteres dient dazu, unterschiedliche Gefüge sowie verschiedene Bedingungen in Paläooberflächen zu unterscheiden, z. B. Kryoturbation und fluviale Strukturen differenzieren. Die Einregelung der Längsachsen der Körner (rund 50) ergeben die vorzeitlichen Bedingungen der Sedimentation.

\section{Sitummetrie und Poldiagramme}

Paläoklimatische Phänomene wie z. B. Kryoturbationen oder sedimentäre Strukturen fluvialen Ursprungs lassen sich anhand der sitummetrischen Analyse und Poldiagramme folgender Gefüge erkennen: 


\section{Profil Liss 20-H}

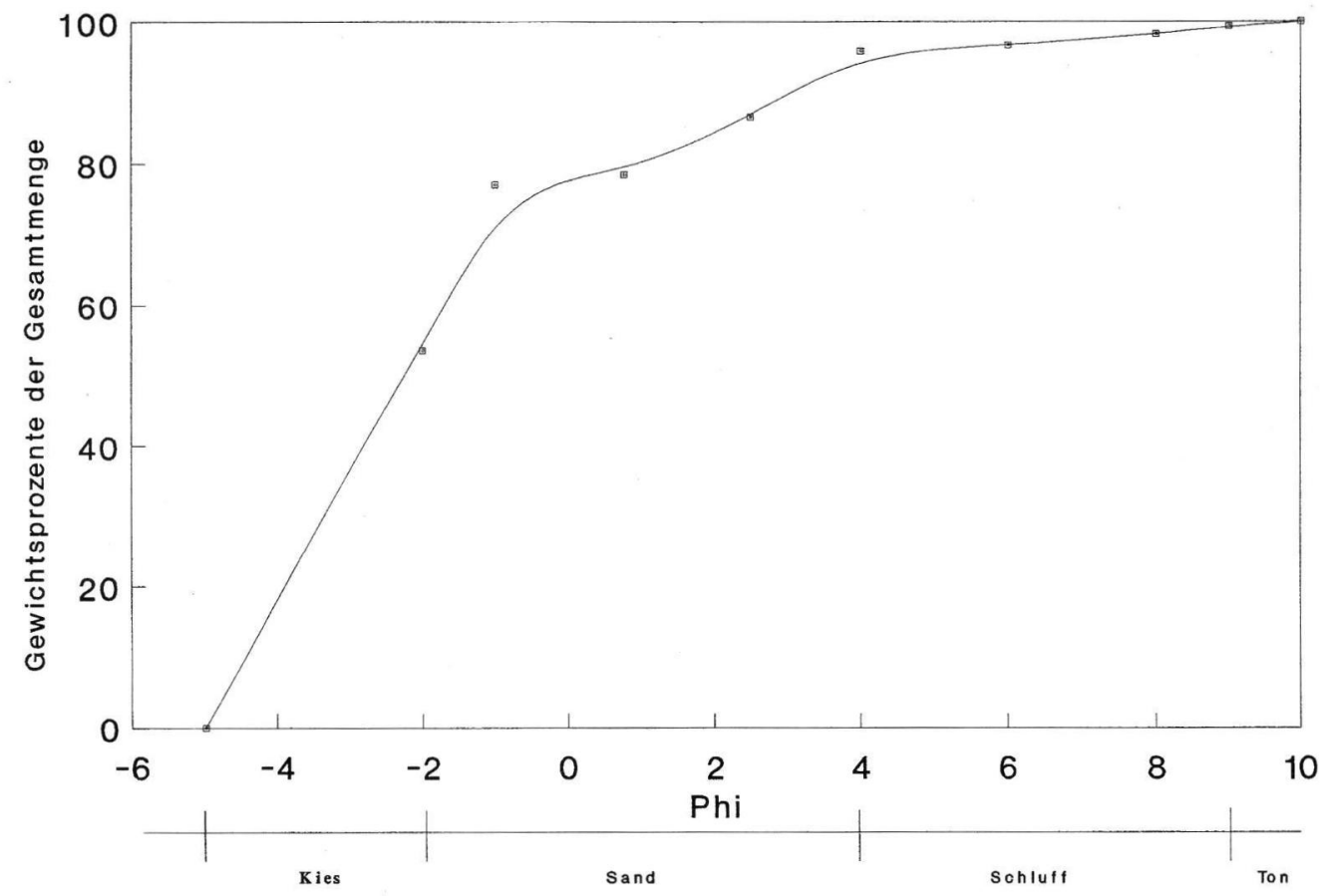

Abb. 9: Profil Liss. Körnungskurven des Füllmaterials eines syngenetischen fossilen Eiskeils und eines Fensters.

Fig. 9: Profile Liss. Cumulative frequency curve for the filling of a syngenetic ice wedge cast and a window.

\subsection{Profil Liss}

(Abb. 3 und dazugehörige Diagramme)

Durch die Sitummetrie und die Analyse von Poldiagrammen kann man die vorzeitlichen Bedingungen rekonstruieren. Abb. 3 zeigt folgende Schwerpunkte der sitummetrischen Analyse:

0- Sektor „Pseudomorphose“ (Abb. 5: 0): Maximum (42\%) in der Gruppe 4 kennzeichnet eine Kollapsstruktur in Verbindung mit einer kryogenen Struktur. Das Poldiagramm zeigt eine eindeutige Konzentration im Zentrum an, die eine deutliche Tendenz der Klasten zur Vertikalität definiert. Kollapsstrukturen in aktuellen Milieus aus der "Cordillera Frontal“ wurden von Твомвотто (1990) beschrieben. Die Kollapsstruktur entsteht, wenn Gerölle in kryogene Risse fallen und/oder wenn sich längliche RP durch die Bewegung, die eine kryogene Entspannung auslöst, vertikal anordnen. Die übrigen Pole befinden sich in einer Zone mit NE-Orientierung und Pseudovertikalität (sie bewegen sich vom Äußeren zum Inneren des Kreises).
1- Sektor „Oberes Fenster": Polymodalität. Gewisse Präferenz bei 3I (27\%). Das Poldiagramm zeigt Bimodalität: eine Gruppe mit NE-Orientierung und eine Gruppe mit E-NE-Orientierung.

2- Sektor "Säule": Maximum (37\%) bei 2D. Minimum $(6 \%)$ bei 3D. Die übrigen Werte sind etwa gleichmäßig auf die anderen Gruppen verteilt bzw. in den Gruppen auf der linken Seite etwas stärker vertreten. Das Poldiagramm zeigt eine Variation der regionalen Tendenz Richtung Osten. Das könnte mit der Aktivität der Verfüllung und den mineralisierenden Fließbewegungen zusammenhängen, die durch die Hangneigung bedingt sind.

3- Sektor „Linkes Fenster“: Polymodalität. Geringe Überzahl bei $1 \mathrm{C}(25 \%)$. Das Poldiagramm in diesem Teil des „Fensters“ weist eine Disposition in E-SERichtung auf.

4- Sektor „Nougat“: Polymodalität. Gewisse Präferenz bei 3D (23\%). Das Poldiagramm zeigt eine deutliche Tendenz Richtung E-NE auf. Die kryogene 


\section{Sektor: "Säule"}
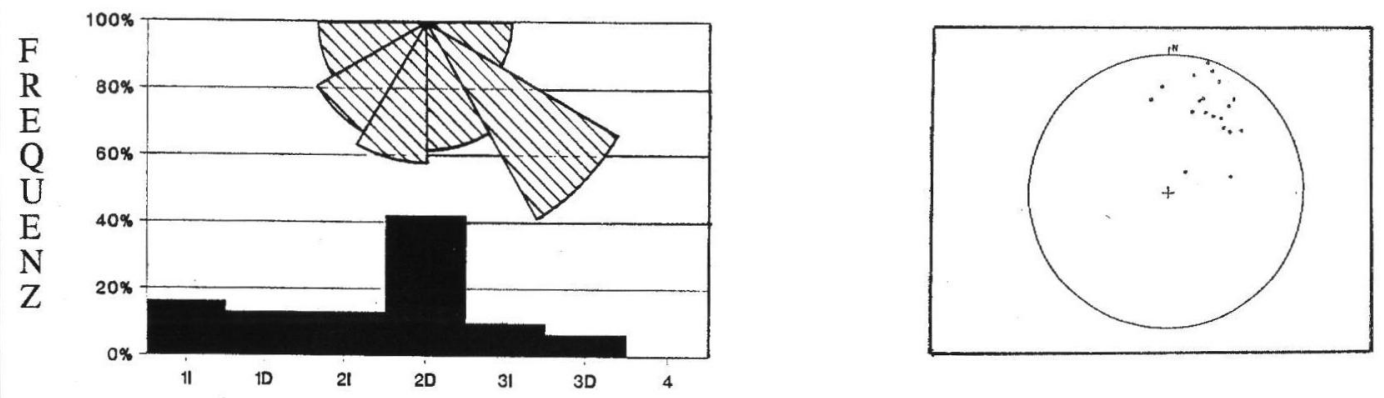

\section{ORIENTIERUNG}

Sektor: "Linkes Fenster"
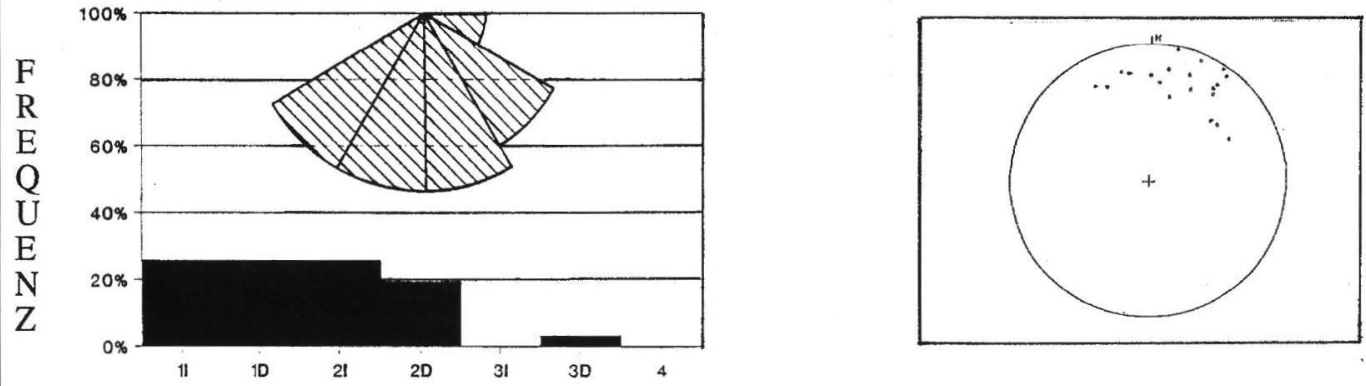

ORIENTIERUNG

Sektor: "Rechtes Fenster"
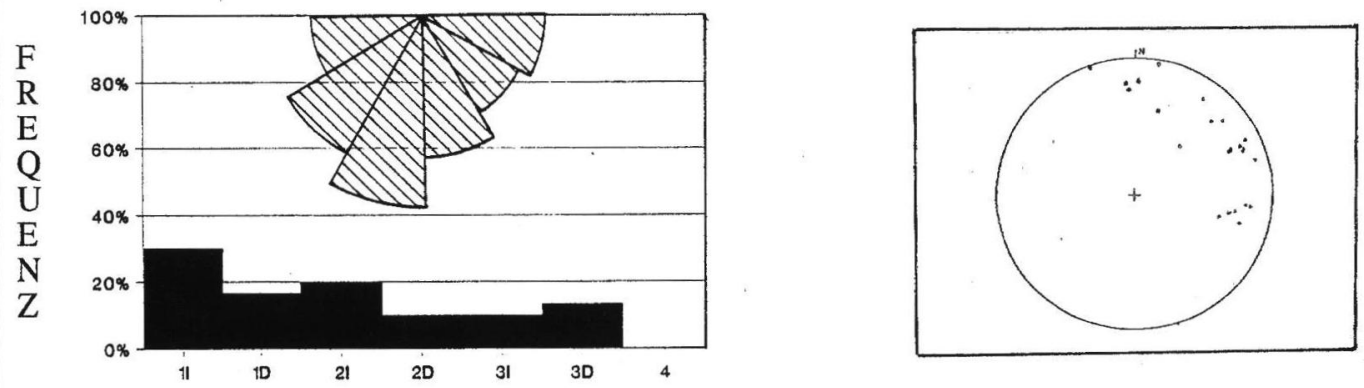

\section{ORIENTIERUNG}

Abb. 10: Profil Andermatten. Sitummetrie und Poldiagramme.

Fig. 10: Profile Andermatten. Sitummetry and pole-diagrams.

Beeinflussung (Solifluktion und/oder Kryoturbation) dieser Schicht oberhalb der RP ist möglicherweise die Ursache sitummetrischer Heterogenität.

5- Sektor „Rechtes Fenster“: Maximum bei 1I (34\%). Gute Aufteilung der Orientierung auf die übrigen
Gruppen. Das Poldiagramm in diesem Sektor weist eine eindeutig definierte Disposition in E-SE-Richtung auf.

6-Sektor „Rechte Apophyse“: Maximum bei 2I (48\%). Das Poldiagramm zeigt eine Hauptausrichtung nach NO auf. 


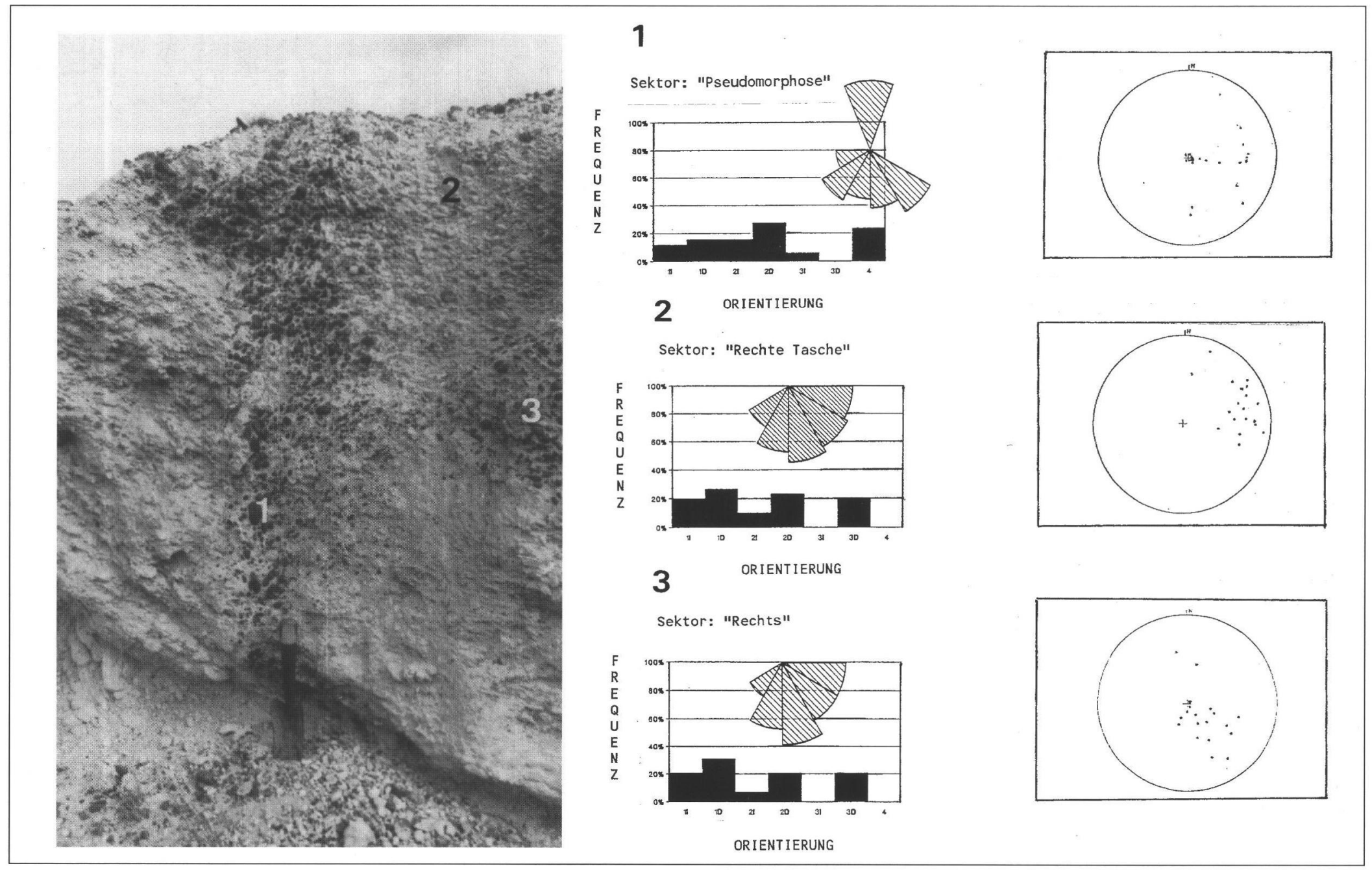




\subsection{Interpretation des Profils Liss}

Die syngenetischen Eiskeile entstehen durch die Sedimentation der patagonischen Gerölle. Abb. 9 zeigt die Textur des Eiskeils anhand der Distribution der Gewichtsmenge in Prozentanteilen. Die Analyse der granulometrischen Verteilung $(<20 \mathrm{~mm})$ und die statistischen Daten, die die Füllung der Eiskeil-Pseudomorphose kennzeichnen, ergeben eine leptokurtische Kurve mit einer Asymmetrie von 0,2 - 0,3 bei sehr geringer Standardabweichung. Der Mittelwert und der Median entsprechen der granulometrischen Fraktion des Sandkorns. Die Untersuchung des Materials $<2 \mathrm{~mm}$ ergibt einen Hauptanteil an Feinsand (Твомвотто 1992). Schluff und Ton zusammengenommen machen $4,2 \%$ aus (davon 3,5\% Schluff). Ein beispielhaftes Fenster zeigt dagegen eine andere Körnungslinie, wobei Schluff und Ton unwichtig sind und Feinsand nur rund $4 \%$ beträgt. Die Fenster konservieren andererseits die ursprüngliche fluviale Struktur. Material $<0.063$ enthalten die Fenster kaum. Die kryogene Aktivität verursacht eine besondere Struktur aus Kies, die mehr Feinsedimente konserviert. Die kryogene Struktur hat eine Taschen-Funktion. Sie verschluckt Feinsedimente, die danach relevant für die Kalkablagerung sind. Durch die Sitummetrie und die Poldiagramme kann man diese kryogenen Strukturen allerdings erkennen.

Aus dem oben Gesagten läßt sich folgendes schließen:

a) Der fossile Eiskeil läßt sich eindeutig identifizieren, weil auch die Eigenschaften der Diagramme die Tendenz einer Vertikalisierung der Klasten nachweisen.

b) Die große Vielfalt der Orientierung der Klasten ist der Grund für die Polymodalität. Sie läßt sich möglicherweise auf die Überlappungen während der $\mathrm{Ab}$ lagerung der Gerölle und/oder auf die Störungen, die durch Gefrieren und Tauen hervorgerufen wurden, zurückführen.

c) Der untere Abschnitt des Profils Liss weist gewisse Unterschiede auf. Wenn man von einer allgemeinen Fließrichtung in NE-Richtung ausgeht (siehe Grober 1952; Haller 1981), lassen sich Unterschiede bei den unteren „Fenstern" (Abb. 3: 3 und 5), sowie in der Apophyse (6) feststellen, wo die Frequenzen mit einer E-SE-Orientierung zusammenhängen. Das legt den Gedanken nahe, daß die Gerölle von einer anderen Paläooberfläche oder von einer lokalen Variation der Hangneigung beeinflußt sein können. Zeichnet sich schon der "Bajo Grande“ ab (Abb. 1)? d) Man kann auch davon ausgehen, daß die Risse, Öffnungen in der Paläooberfläche und die kalkhaltigen Lösungen mit ihrer späteren Mineralisierung dazu beigetragen haben, daß sich die ursprüngliche Disposition der Klasten geändert hat.

\subsection{Interpretation der anderen Profile}

\subsubsection{Profil Andermatten (Abb. 10)}

Das Profil Andermatten (Abb. 1) ist ein typisches RPProfil mit kalkhaltigem Netz. Die sitummetrische Analyse der Säule läßt keine Kollapsstrukturen oder periglaziale Strukturen erkennen. Trotz der Ähnlichkeit mit dem vorherigen Profil ist dieses nach $W$ ausgerichtet. Die Orientierungen der Längsachsen (Abb. 10) zeigen ein Maximum bei 2D (42\%) und ein Minimum bei 3D (6\%). Während ein gewisses Übergewicht der Orientierungen der Gruppen sich auf der linken Seite befindet, zeigt das Poldiagramm eine regionale Tendenz in N-NE-Richtung. Die sitummetrische Analyse der Fenster ergibt Polymodalität, obwohl auf dem rechten Fenster eine gewisse Präferenz bei 1I (30 \%) auffällt. Die Poldiagramme des rechten Fensters bestätigen Polymodalität bei 2 und 3.

In allen drei Fällen (Abb. 10) und unter Berüicksichtigung des Grundwassers würden die Orientierungen eine Fließrichtung nach NE andeuten. Während sich innerhalb der "Fenster" die Ablagerungsstrukturen nicht verändern, würden die „Säulen“ eine Schwachstelle oder einen Riß darstellen, je nach Fließrichtung und Gefälle im allgemeinen.

\subsubsection{Profil Vallejo (Abb. 11 und dazugehörige Diagramme)}

Anhand der sitummetrischen Analyse und der Poldiagramme des Profiles Vallejo läßt sich recht einfach ein fossiler Eiskeil erkennen. Das Maximum bei 2D (Abb. 11: 1) im pseudomorphen Sektor könnte mit der ursprünglichen Struktur zusammenhängen. Die Fälle 2 und 3 sind sehr ähnlich und bestärken die regionale Fließrichtung nach NE.

Die Merkmale der Sektoren (Abb. 11) zeigen folgende Schwerpunkte:

1- Sektor "Pseudomorphose": Maximum bei 2D (27 \%) und in der Gruppe 4 (24\%). Das Poldiagramm ergibt eine vertikale Sortierung der Klasten und zwei Gruppen mit vertikaler Tendenz.

2- Sektor „Rechte Tasche“: Maximum bei 1D (27\%) und bei 2D (23\%). Bei 3I ist der Wert 0. Das Poldiagramm zeigt einen Nukleus der Vertikalität der Klasten, der eine E-SE orientierte Gruppe anzeigt.

3- Sektor „Rechts“: Maximum bei 1D (31\%). Bei 2D sowie 3D sind die Werte gemäßigt (21\%); bei 3I liegt der Wert bei 0 . Das Poldiagramm verhält sich wie im vorherigen Fall. 


\section{Schlußfolgerungen}

1. Kalte Phänomene, die unter dem Begriff „Penfordd Kryomer" zusammengefaßt werden, haben die RP während und nach ihrer Ablagerung beeinflußt. Sie sind sehr alt, weil sie syngenetische fossile Eiskeile enthalten.

2. Die kältesten glazialen Impulse mit Permafrost in Patagonien, sind die kryogenen Verursacher der thermischen Kontraktionen und der syngenetischen und epigenetischen Eiskeile in den RP-Profilen.

3. Verschiedene kryogene Phasen führen zu einer fluktuierenden Gefrier- und Auftauschicht und zu Austrocknungsprozessen. Dadurch läßt sich das Aufreißen in fein krackelierte Netze erklären, die das Zirkulieren mineralisierenden Wassers ermöglichen.

4. „Säulen“ und quasihorizontale Schichten, die a posteriori mit den verschiedenen Karbonatisierungsablagerungen in Verbindung treten, stellen die Zirkulationswege und Risse dar, die die ursprüngliche Textur der RP modifiziert haben. Die Mineralisierung führt zu einer veränderten Orientierung der Klasten. Die „Fenster“, die möglicherweise langsamer auftauen, stellen vorübergehende Freiräume ohne bedeutende Kalkablagerung dar.

5. Die so entstandenen Kalkrete waren ariden Phasen unterworfen und lassen sich mit Texturveränderungen in den RP in Verbindung bringen, die eine Karbonatisierung gefördert haben.

6. Die RP-Profile zeigen deutlich zwei morphologisch sehr verschiedene Schichten. Die obere Schicht weist bedeutende texturelle Veränderungen und eine ungeordnete Struktur auf, die sich auf periglaziale Phänomene zurückführen lassen könnten. Die untere Schicht zeigt eine tendenzielle E und NEOrientierung der Klasten.

7. Die vorwiegende E-NE-Orientierung variiert im unteren, mit RP verfülltem Teil der Senken aufgrund lokaler Variationen der Hangneigung.

8. Die untere RP-Schicht mit den charakteristischen „Säulen“ und „Fenstern“ weist Variationen bei der Orientierung der Klasten auf, die mit den Netzen zusammenhängen, die in der Paläo-Auftauschicht oder der Schicht des Gefrierens, Auftauens und Austrocknens entstehen.

9. Die Klasten der Eiskeilpseudomorphosen haben eine Tendenz zur Vertikalität und lassen sich eindeutig in den Diagrammen mit Hilfe der angewandten Techniken unterscheiden.

\section{Dank}

Die vorliegende Arbeit konnte dank der finanziellen Unterstützung von CONICET (PIA 0122/90) durchgeführt werden. Ich möchte mich außerdem bei Chem. Ing. Héctor Alcántara und ALUAR dafür bedanken, daß mir Labors und das Rechenzentrum zur Verfügung gestellt wurden. Darüber hinaus bin ich Prof. Dr. Dietrich Barsch (Universität Heidelberg), Dr. Gerardo Bossi (Sedimentologisches Institut der Universität Tucumán), meiner Frau Sabine für die Lektüre des déutschen Manuskripts, Fernando Jaguer, Lori Taylor und Mateo Cornejo (Cenpat) zu Dank verpflichtet.

\section{Schriftenverzeichnis}

Ahumada, A.-L. \& Trombotto, D. (1984): Estudios Periglaciales en la Lagunita del Plata, Provincia de Mendoza. Noveno Congreso Geológico Argentino. S. C. de Bariloche, Actas IV: 22-34, 12 Abb.; Buenos Aires.

Auer, V. (1956): The Pleistocene of Fuego-Patagonia. Part I: The Ice and Interglacial Ages. Annales Academiae Scientiarum Fennicae, III. Geologica-Geographica, 45: 226 pp., 189 figures, 5 appendices; Helsinki.

- (1970): The Pleistocene of Fuego-Patagonia. Part V: Quaternary Problems of Southern South America. Annales Academiae Scientiarum Fennicae, III. GeologicaGeographica 100: 194 pp.; Helsinki.

Beltramone, C. A. (1989): Observations of fossil ice wedges corresponding to two cryogenic periods in Puerto Madryn surrounding areas. IGCP Project Nr. 297: "Geocryology of the Americas", Abstracts and Reports: 2323; Mendoza.

Bronge, Ch. (1992): Holocene climatic record from lacustrine sediments in a freshwater lake in the Vestfold Hills, Antarctica. Geografiska Annaler 74 A: 47-58; Stockholm.

CABrera, A. (1976): Regiones Fitogeográficas Argentinas. Enciclopedia Argentina de Agricultura y Jardineria, Tomo II, 1: 85 ps.; Buenos Aires.

Caldenius, C. (1940): The Tehuelche or Patagonian Shingle-Formation. Geografiska Annaler 22: 160-181; Stockholm.

Clapperton, C. (1983): The Glaciation of the Andes. Quaternary Science Reviews, 2: 83-155.

- \& RoBERTS, D. (1986): Quaternary sea level changes in the Falkland Islands. Quaternary of South America and Antarctic Peninsula, 4: 99-117.

Codignotto, J. O., KoкоT, R. R. \& Marcomini, S. C. (1991): Neotectonism and Sea-Level Changes in the Coastal Zone of Argentina. Journal of Coastal Research, 8: 125133; Charlottesville, Virginia.

CORTE, A. (1991): Chronostratigraphic Correlations of Cryogenic Episodes in Central Andes and Patagonia. Permafrost and Periglacial Processes, 2: 67-70.

- \& Beltramone, C. (1984): Edad de las Estructuras Geocriogénicas de Puerto Madryn, Chubut, Argentina. Acta Geocriogénica, 2: 67-72; Mendoza.

CZAJKA, W. (1955): Rezente und pleistozäne Verbreitung und Typen des periglazialen Denudationszyklus in Argentinien. Acta Geographica 14, 10: 121-140; Helsinki.

Del Valle, H. F. \& Beltramone, C. A. (1987): Morfología de las Acumulaciones Calcáreas en algunos Paleosuelos 
de Patagonia Oriental (Chubut). Ciencia del Suelo, 5: 77-87.

Feruglio, E. (1950): Descripción Geológica de la Patagonia. Tomo III: 431 ps, YPF; Buenos Aires.

GARlefF, K. \& StINGL, H. (1984): Neue Befunde zur jungquartären Vergletscherung in Cuyo und Patagonien. Berliner Geogr. Abh., 36: 105-112, 3 Abb.; Berlin.

- (1985): Jungquartäre Klimageschichte und ihre Indikatoren in Südamerika. Zbl. Geol. Paläont., Teil I, 11/12: 1769-1775, 1 Abb., 1 Tabelle; Stuttgart.

Gonzalez, M.-A. (1989): Holocene Levels in the Bahía Blanca Estuary, Argentine Republic. Jour. of Coastal Research, 5: 65-77; Charlottesville, Virginia.

- \& Musacchio, E., Garcia, A., Pascual, R. \& Corte, A. (1981): Las líneas de costa (Holoceno) de la Salina del Bebedero (San Luis, Argentina). Implicancias paleoambientales de sus microfósiles. VIII Congreso Geológico Argentino, San Luis. Actas III: 617-628; Buenos Aires.

- \& TrombotTo, D. (1990): Neogene Sand Desert Development During Global Cooling Episodes in Southern South America. Fundación Carl C:zon Caldenius, Notas Técnicas y Cientificas, 3: 10 ps.; Buenos Aires.

Groeber, P. (1950): Quartäre Vereisung Nordpatagoniens. Sonderdruck der Zeitschrift „Südamerika“: 6 S.; Buenos Aires.

- (1952): Glacial, Tardío y Post-Glacial en Patagonia. Revista del Museo Municipal de Ciencias Naturales y Tradicional de Mar del Plata, 1: 79-103.

Haller, M. J. (1981): Descripción Geológica de la Hoja 43h - Puerto Madryn. Servicio Geológico Nacional, Boletin Nr. 184: 41 ps.

HALlet, B. (1976): Deposits formed by subglacial precipitation of $\mathrm{CaCO}_{3}$. Geological Society of America Bulletin, 87: 1003-1015.

Heusser, C. \& RABASSA, J. (1987): Cold climatic episode of Younger Dryas age in Tierra del Fuego. Nature, 328: 609-611.

Liss, C. Ch. (1969): Fossile Eiskeile (?) an der Patagonischen Atlantikküste, Zeitschrift für Geomorphologie, Neue Folge, 13: 109-114, 5 Abb.; 1 Berlin - 7 Stuttgart.

LÜTTIG, G. (1965): Interglacial and Interstadial Periods. J. Geol. 73: 579-591.

Mercer, J. H. (1976): Glacial History of Southernmost South America. Quaternary Research 6: 125-166; Washington.

- (1985): Las variaciones glaciares del antiguo Cenozoico en Sudamérica, al sud del Ecuador. Acta Geocriogénica 3: 86-105; Mendoza.

- \& SuTter, J. F. (1982): Late Miocene-Earliest Pliocene Glaciation in Southern Argentina: Implications for
Global Ice-Sheet History. Palaeogeography, Palaeoclimatology, Palaeoecology, 38: 185-206; Amsterdam.

- \& AGER, T. (1983): Glacial and Floral Changes in Southern Argentina. nat. Geogr. Soc. Res. Reports 15: 457-477.

Nilsson, T. (1983): The Pleistocene. Enke: 651 pp., 292 figures, 23 stratigraphical tables; Stuttgart.

PetTijohn, F. J., PoTter, P. E. \& Siever, R. (1987): Sand and Sandstone. Springer Verlag: 553 pp., 355 figures; New York.

PÉWÉ, T. L. (1984): Deposition of Windblown Dust in Central Arizona, USA. En M. Pécsi (ed), Lithology and Stratigraphy of Loess and Paleosols, Hungarian Academy of Sciences: 305-325; Budapest.

Rabassa, J., Bujalesky, G.; Meglioli, A., Coronato, A., Gordillo, S., Roig, C. \& Salemme, M. (1992): The Quarternary of Tierra del Fuego, Argentina: the status of our knowledge. Sveriges Geologiska Undersökning, Ser. Ca 81: 249-256.

Schobinger, J. (1973): Prehistoria de Suramérica. Editorial Labor: 296 ps.; Barcelona.

Stine, S. \& STINE, M. (1990): A record from Lake Cardiel of climate change in southern South America. Nature, 345: 705-707.

SYLWAN, C. (1989): Paleomagnetism, Paleoclimate and Chronology of Late Cenozoic Deposits in Southern Argentina. Meddelanden fràm Stockholms Universtets Geologiska Institution, 277: 110 pp.; Stockholm.

Troll, C. \& PAfFEN, K. H. (1969): Karte der Jahreszeiten-Klimate der Erde. Erdkunde, XVIII: 28 S., 15 Abb., 1 Karte, 1 Legende.

TrombotTo, D. (1992): The Cryomere Penfordd, Patagonia. International Workshop: „Permafrost and Periglacial Environments in Mountain Areas", 3rd. Meeting IGCP Project 297: "Geocryology of the Americas": 33 pp., 23 Abb.; Calgary.

- \& STEIN, B. (1993): El último criómero pleistocénico en la región de los „kopjes“ y de las grandes mesetas, Patagonia, Argentina. XII Congreso Geológico Argentino, Mendoza, Actas, Vol. VI: 92-96, 5 Abb.; Buenos Aires.

VillalbA, R. (1990): Climatic fluctuations in Northern Patagonia during the last 1000 years as inferred from treering records. Quaternary Research 34: 346-360.

Vogt, T. (1990): Cryogenic Physico-chemical Precipitations: Iron, Silica, Calcium Carbonate. Permafrost and Periglacial Processes, 1: 283-293.

WeILER, N. E. (1988): Depósitos litorales del Pleistoceno tardío y Holoceno en Bahía Anegada, Provincia de Buenos Aires. Segunda Reunión Argentina de Sedimentología, Actas: 245-249; Buenos Aires.

Manuskript eingegangen am 03.12.1993 